\title{
IMPLEMENTATION OF LOW-COMPLEXITY PRINCIPAL COMPONENT ANALYSIS FOR REMOTELY SENSED HYPERSPECTRAL-IMAGE COMPRESSION
}

\author{
Qian Du, Wei Zhu, and James E. Fowler \\ Department of Electrical and Computer Engineering \\ GeoResources Institute \\ Mississippi State University, USA
}

\begin{abstract}
Remotely sensed hyperspectral imagery has vast data volume, for which data compression is a necessary processing step. Spectral decorrelation is critical to successful hyperspectral-image compression. Principal component analysis (PCA) is well-known for its superior performance in data decorrelation, and it has been demonstrated that using PCA for spectral decorrelation can yield rate-distortion and data-analysis performance superior to other widely used approaches, such as the discrete wavelet transform (DWT). However, PCA is a datadependent transform, and its complicated implementation in hardware hinders its use in practice. In this paper, schemes for low-complexity PCA are discussed, including spatial down-sampling, the use of non-zero mean data, and the adoption of a simple PCA neural-network. System-design issues are also investigated. Experimental results focused on the fidelity of pixel values and pixel spectral signatures demonstrate that the proposed schemes achieve a trade-off between compression performance and system-design complexity.
\end{abstract}

Index Terms - Hyperspectral-image compression, principal component analysis (PCA), JPEG2000, discrete wavelet transform (DWT)

\section{INTRODUCTION}

A hyperspectral imaging sensor collects data in hundreds of contiguous spectral bands with very narrow bandwidth over the same spatial area on the Earth. As a relatively new technique in remote sensing, hyperspectral imaging has attracted great interest due to its potential to provide more powerful diagnosis in material detection, classification, and quantification than traditional multispectral imaging. The resulting three-dimensional (3D) hyperspectral image cube typically has a very large data volume. For instance, each frame from the Airborne Visible/InfraRed Imaging Spectrometer (AVIRIS) has 224 bands with $512 \times 614$ pixels in each band. Using 16 bits to represent each pixel element, the total data size is more than 100 Mbytes. When the aerial platform is operated at an altitude of $20 \mathrm{~km}$, the spatial resolution is about $20 \mathrm{~m}$, and the image covers an area of approximately $125 \mathrm{~km}^{2}$. When the area to be covered is larger, the data size increases dramatically.

In addition to high spatial correlation, a hyperspectral image also has high spectral correlation. Consequently, a data-compression algorithm should perform both spatial and spectral decorrelation efficiently. Wavelet-based lossycompression techniques are of interest because of their excellent rate-distortion performance. In addition to JPEG2000, the most widely known wavelet-based compression techniques include set partitioning in hierarchical trees (SPIHT) [1] and set-partitioned embedded block (SPECK) coder [2]. SPIHT and SPECK have been extended to 3D-SPIHT [3] and 3D-SPECK [4] for hyperspectral-image compression, respectively. JPEG2000 has also been used for 3D hyperspectral-image coding [5-6]. In these 3D DWT-based algorithms, the performance is best when spectral decorrelation is conducted separately from spatial decorrelation [7] in the form of a wavelet-packet transform consisting of a 1D spectral DWT followed by a 2D spatial DWT. In [8], it was shown that rate-distortion performance can be significantly improved by employing principal component analysis (PCA), a well-known datadecorrelation method, for spectral decorrelation. In [8], the resulting algorithm is referred to as PCA+JPEG2000, which is distinct from DWT+JPEG2000 wherein a DWT is used for spectral decorrelation. This observation further demonstrates that efficient spectral decorrelation is critical for hyperspectral-image compression.

When only a subset of principal components (PCs) is preserved for compression, the rate-distortion performance can be further improved [8] as opposed to the case in which all PCs are retained and coded. It is argued that this phenomenon arises from the fact that the minor PCs contain mostly noise only, and it is better to assign zero bits to them so as to reserve more bits for the major PCs in bit allocation. In fact, in the JPEG2000 bitstream, header information consumes bitrate for each $\mathrm{PC}$ in the bitstream, even if no bits are assigned to a given $\mathrm{PC}$, which wastes additional bits. We refer to PCA retaining only a subset of PCs employed in conjunction with JPEG2000 as SubPCA+JPEG2000. 
Fig. 1 presents the rate-distortion performance of the aforementioned algorithms when encoding the 16-bit AVIRIS Jasper Ridge radiance dataset. The detailed SNR values in $\mathrm{dB}$ versus bitrates in bits per pixel per band (bpppb) are listed in Table I. We see that PCA+JPEG2000 brings about a 5-10 $\mathrm{dB}$ increase in SNR as compared to DWT+JPEG2000, whose SNR in turn is about $0.5 \mathrm{~dB}$ greater than those of 3D-SPIHT and 3D-SPECK. SubPCA+JPEG2000 further enhances performance, particularly for low bitrates. Fig. 2 shows how the ratedistortion changes with the number of PCs maintained for compression when using SubPCA+JPEG200. We see that the maximum SNR does not occur when all $224 \mathrm{PCs}$ are used and that the SNR improvement is more significant for low bitrates. As a consequence of these observations, for the remainder of this paper, we focus on SubPCA+JPEG2000 using, for each bitrate under consideration, the number of PCs producing maximum SNR for that bitrate. With this coder, we examine implementation of low-complexity PCA and related system-design issues.

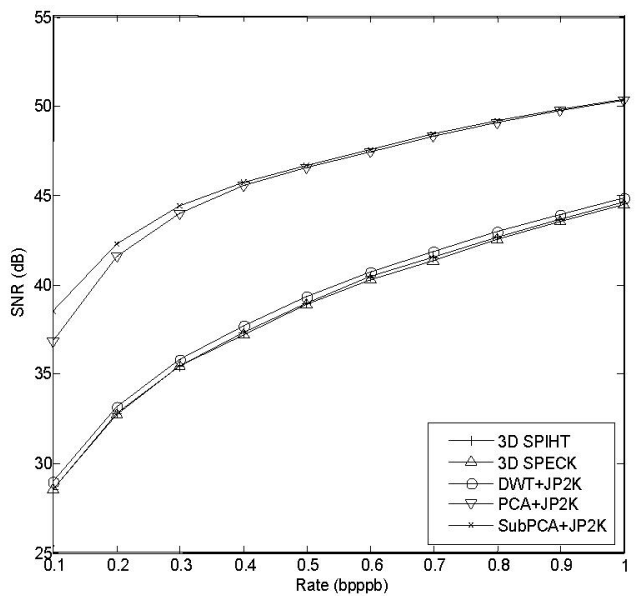

Fig. 1. The rate-distortion performance using different algorithms to encode the Jasper Ridge radiance dataset.

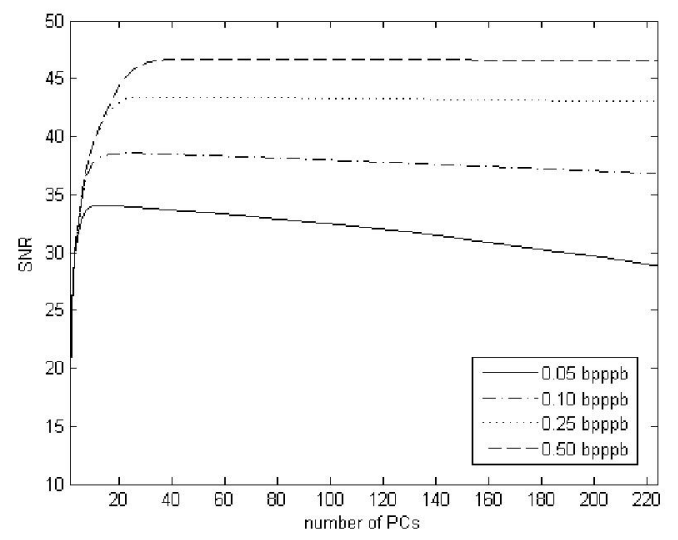

Fig. 2. SNR vs. the number of $P C$ s retained when using SubPCA + JPEG2000 to encode the Jasper Ridge radiance dataset.

\section{LOW-COMPLEXITY PRINCIPAL COMPONENT ANALYSIS}

\subsection{Computational complexity of PCA}

When performing PCA, the data mean is estimated and subtracted. Then the key procedures of PCA follow which include estimating the covariance matrix $\Sigma$ of the whole image, solving eigenvector problems, and computing matrix-vector products, all of which are numerically intensive. Because of the data-dependent nature of PCA, the transform matrix has to be recomputed for each dataset. Given a hyperspectral image with $L$ bands and $N$ pixel vectors, the complexity of PCA comprising the three processes can be calculated as follows. The first process is the estimation of the covariance matrix, wherein the number of multiplications is $L^{2} N$; the second process is the determination of eigenvectors, wherein the typical number of multiplications is on the order of $L^{3}$ with a certain matrixoperation method [9]; and the third process is the computation of transformed pixels, wherein the number of multiplications is $L^{2} N$. When only the first $M(M<L)$ PCs are used in SubPCA+JPEG2000, then the third process has $M L N$ multiplications. If only $M \mathrm{PCs}$ are generated, then the second process involves multiplications on the order of $M L^{2}$. For most hyperspectral datasets, $N$ is much greater than $L$, so the computational complexity is mainly from the first and third processes.

\subsection{Spatial down-sampling for low-complexity PCA}

Since the calculation of the covariance matrix $\Sigma$ requires the largest number of multiplications, complexity can be reduced by using only a small subset of pixels [10]. This spatial down-sampling can be conducted randomly and 1\% of the pixels can provide a very accurate estimation of the covariance matrix [10]. Such subsampling will reduce the number of multiplications in the first step to $0.01 L^{2} N$. The resulting compression scheme is denoted with a "RDS" prefix, standing for Random Down-Sampling.

\subsection{Non-zero mean data for low-complexity PCA}

In many cases, whether or not the mean is removed from the data preceding eigen-decomposition does not affect the final results of PCA. If the mean is not removed (i.e., non-zero mean data), then additional computations can be saved in mean estimation and subtraction. In other words, the correlation matrix $\mathbf{R}$ rather than the covariance matrix $\Sigma$ is used in PCA. This reduces complexity since neither addition nor subtraction are needed for mean estimation which, in turn, greatly simplifies hardware design and implementation. The resulting compression scheme is denoted with a "NZM" prefix, standing for Non-Zero Mean data. 


\subsection{A simple neural network for low-complexity PCA}

Neural-network (NN) approaches have been developed for PCA [11]; such schemes apply a learning rule to update the estimate of an eigenvector without the need of explicitly calculating the covariance matrix $\Sigma$. In [12], we proposed a simple neural network with one output neuron and $L$ input neurons, with the weight vector $\mathbf{w}$ being updated according to a Hebbian learning rule. When the neural network converges, $\mathbf{w}$ approaches the first eigenvector and the output, $y$, approaches the first eigenvalue. Denote the input as $\mathbf{x}$ (a pixel vector from the hyperspectral image) and the output as $y=\mathbf{w}^{T} \mathbf{x}$. According to Hebbian learning, $\Delta \mathbf{w}=\alpha y \mathbf{x}$, where $\alpha$ is the learning rate; for simplicity here, we set $\alpha=1$. The weight vector needs to be normalized to prevent unlimited growth. Therefore, the additional equations for the updates are: $\mathbf{w} \leftarrow \mathbf{w}+\Delta \mathbf{w}$ and $\mathbf{w} \leftarrow \mathbf{w} /\|\mathbf{w}\|$. Both sequential and batch modes can be used for learning. In the sequential mode, $\mathbf{w}$ is adjusted after each pixel is presented to the network. On the other hand, in the batch mode, $\mathbf{w}$ is not updated until after all pixels from the image are presented to the network. We find that batch mode provides more accurate results; i.e., the final result is closer to the actual eigenvector. As a consequence, we consider batch-mode training exclusively in the following.

After the first eigenvector $\mathbf{w}_{1}$ is estimated, the second eigenvector $\mathbf{w}_{2}$ can be determined similarly. However, the dataset is first projected onto the subspace constructed by the first eigenvector $\mathbf{w}_{1}$, and the projection is removed from the data before $\mathbf{w}_{2}$ is determined. Likewise, to estimate eigenvector $i, \mathbf{w}_{i}$, projections along the previous $i-1$ eigenvectors are removed before the data is presented to the neural network. In other words, the data update equation when estimating $\mathbf{w}_{i}$ for any $i>1$ is $\mathbf{x} \leftarrow \mathbf{x}-\mathbf{w}_{i-1} \mathbf{w}_{i-1}^{T} \mathbf{x}$. This process, similar to Gram-Schmidt orthogonalization, guarantees the extraction of additional orthogonal eigenvectors. Each weight vector can be randomly initialized. In order to increase the convergence speed, a random initialization can be made orthogonal to the previously determined eigenvectors. The detailed algorithm can be found in [12].

It should be noted that this one-output neural network finds eigenvectors one after another. Data orthogonalization is achieved by simple matrix multiplication outside the neural network. The generalized Hebbian learning algorithm in [13] uses multiple outputs to find multiple eigenvectors simultaneously, and its weight update equation includes the term related to data orthogonalization. There are two advantages of our algorithm: 1) it is more flexible in generating any number of eigenvectors as needed because of its sequential nature; and 2) it requires fewer multiplications since the data orthogonalization is accomplished in a single step.

The computational complexity of NN-based PCA increases linearly with the number of pixels $N^{\prime}$ used for training, the number of bands $L$, the number of eigenvectors $M$ to be determined, and the number of iterations $K$ for determining each weight vector. Obviously, it is advantageous to deploy this NN-based approach when using SubPCA+JPEG2000, particularly for low bitrates, since $M$ is small. As for $K$, it can be determined by a user-defined accuracy because a larger $K$ corresponds to a closer estimate of an eigenvector. It has been observed that the value of $K$ can be typically chosen as small as 5 without introducing much degradation to the performance of the spectral decorrelation

NN-based PCA is preferable due to its simple hardware implementation and the high potential for parallelization [11]. In next section, the design of a system including these schemes for low-complexity PCA will be discussed.

\section{IMPLEMENTATION OF LOW-COMPLEXITY PRINCIPAL COMPONENT ANALYSIS}

\subsection{System overview}

As illustrated in Fig. 3, the transform matrix of PCA is sent to the JPEG2000 coder, the encoder applies the spectral transform (reducing the dimensionality as well in the case of SubPCA+JPEG2000), and then the inverse-transform matrix is embedded into the bitstream. The encoder automatically allocates rate simultaneously across the PCs to be coded; i.e., the post-compression rate-distortion (PCRD) process (see, e.g., [5]) is applied to all codeblocks in all PCs, optimally truncating the bitstream for each codeblock. This process produces, in theory, an optimal rate allocation yielding maximum SNR. In the reconstruction process, the decoder automatically extracts the inversetransform matrix and applies it appropriately after the bitstream has been decoded. The implementation of JPEG2000 has been widely studied, such as in [14-16]. As a consequence, we provide here the details for only the implementation of the PCA-based spectral decorrelation.

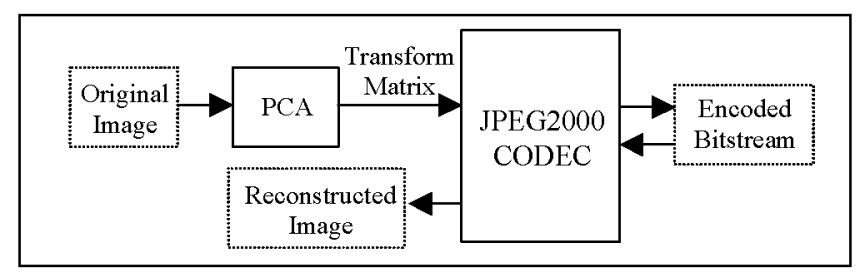

Fig. 3. Block diagram for the overall system.

\subsection{Low-complexity implementation of PCA}

\subsection{Random sampling versus uniform sampling}

The primary route to low-complexity PCA as described in Sec. 2 is spatial down-sampling. If the subsampling is 
performed at random, then a random-number generator needs to be designed using a technique such as in [17-18]. To further simplify the hardware design, uniform downsampling (UDS) can be used instead. For instance, when a sampling ratio $1 \%$ is used, the training set keeps one out of every hundred pixels. This uniform subsampling process is easily accomplished by using a digital counter with the period of 100 . Every time the counter reaches 100 , controlled switches are closed to let the input pixel vector pass. If, on the other hand, the sampling ratio is $2 \%$, the counter period is simply changed to 50 .

This same idea is used for initialization of NN-based PCA, where the initial vectors are not the chosen at random. Rather, a pixel vector is uniformly chosen from the training set as an initial weight. In this case, the counter period is approximately equal to the ratio between the number of pixels in the training sets and the number of PCs to be determined. A block diagram of this low-complexity PCA implementation is illustrated in Fig. 4.

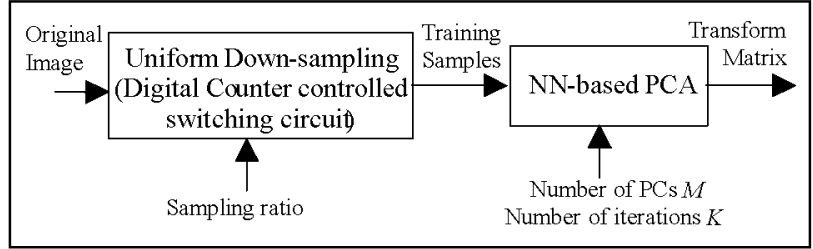

Fig. 4. Block diagram for low-complexity PCA.

\subsubsection{NN-based PCA module}

Fig. 5 shows a block diagram for NN-based PCA. The training samples are projected onto the orthogonal subspace of the previously determined eigenvectors. As mentioned earlier, uniformly selected samples (after orthogonalization) are used as the initial weights. Control circuits check if sufficient iterations are conducted, and if sufficient PCs are generated.

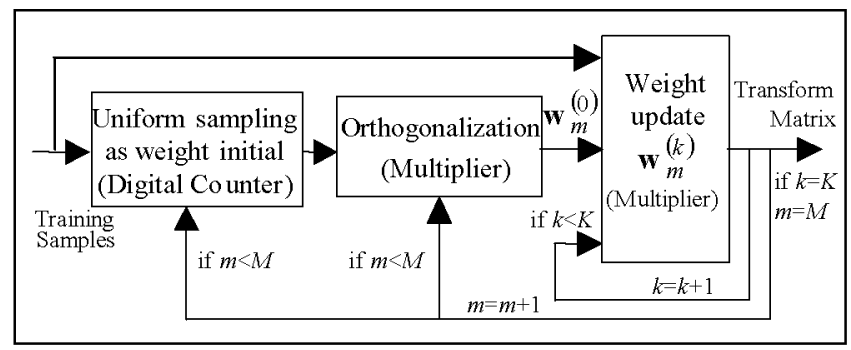

Fig. 5. Block diagram for NN-based PCA.

\section{PERFORMANCE EVALUATION}

Experimental evaluation is conducted to assess the performance of the proposed low-complexity PCA for simplified system design. Table II lists the SNR for 0.1 to 0.5 bpppb when encoding the Jasper Ridge dataset with the original SubPCA+JPEG2000 (using all pixels and singular value decomposition, thereby finding the true eigenvectors); RDS_SubPCA+JPEG200 and UDS_SubPCA+JPEG2000 with $1 \%$ sampling ratio; and RDS_SubPCA_NN+JPEG 200 and UDS_SubPCA_NN+JPEG2000 with the neural network to estimate the eigenvectors (with $K=5$ ). We see that the SNR does not vary much across the various algorithms. Table III lists the SNR when non-zero mean data is used. In this case, the SNR does decrease slightly, and the maximum reduction is about $0.6 \mathrm{~dB}$ at $0.1 \mathrm{bpppb}$. However, the SNR values are still much larger than those of DWT+JPEG2000, 3D SPIHT, and 3D SPECK.

In addition to SNR as a measure of the fidelity of the data, spectral angle mapper (SAM) is also used to measure spectral fidelity, a quality of paramount importance in hyperspectral applications. SAM computes a spectral angle between two pixel vectors - the smaller the spectral angle is, the more similar the two pixels are. The angle between pixel vectors $\mathbf{r}_{1}$ and $\mathbf{r}_{2}$ is calculated $\alpha=\arccos \left(\mathbf{r}_{1}^{T} \mathbf{r}_{2} /\left(\left\|\mathbf{r}_{1}\right\| \cdot\left\|\mathbf{r}_{2}\right\|\right)\right)$. For a hyperspectral image with $N$ pixels, SAM generates $N$ spectral angles. The performance of a compression algorithm can then be evaluated based on the mean, peak value, and standard deviation of the spectralangle matrix. A compression algorithm with smaller mean, peak value, and standard deviation is generally considered to be superior in terms of maintaining spectral fidelity. As listed in Table IV, a DWT for spectral decorreltation (i.e., as in DWT+JPEG2000, 3D SPIHT, and 3D SPECK) yields a much higher spectral discrepancy than does PCA. For the two versions of low-complexity PCA, the performance is similar to that of the original SubPCA+JPEG2000, except that the peak angle becomes somewhat larger (but it is still smaller that those from the DWT-based algorithms).

\section{CONCLUSION}

This paper investigates low-complexity PCA for hyperspectral-image compression. Several mechanisms are proposed for simplifying the implementation, including uniform spatial down-sampling, the use of non-zero mean data, the deployment of a simple neural network with data orthogonalization, and uniform sampling of training samples for algorithm initialization. Experimental results reveal that system simplification does not significantly sacrifice the data-compression performance, either in terms of rate-distortion performance or spectral-angle fidelity. Based on this preliminary work, we hope to study real-time parallel implementations as future work.

\section{REFERENCES}

[1] A. Said and W. A. Pearlman, "A new, fast, and efficient image codec based on set partitioning in hierarchical trees," IEEE Trans. on Circuits and Systems for Video Technology, vol. 6, pp. 243-250, 1996. 
[2] W. A. Pearlman, A. Islam, N. Nagaraj, and A. Said, "Efficient, low-complexity image coding with a setpartitioning embedded block coder," IEEE Trans. on Circuits and Systems for Video Technology, vol. 14, pp. 1219-1235, 2004.

[3] B.-J. Kim, Z. Xiong, and W. A. Pearlman, "Low bit-rate scalable video coding with $3 \mathrm{D}$ set partitioning in hierarchical trees (3D SPIHT)," IEEE Trans. on Circuits and Systems for Video Technology, vol. 10, pp. 1374-1387, 2000.

[4] X. Tang and W. A. Pearlman, "Scalable hyperspectral image coding," Proceedings on IEEE International Conference on Acoustics, Speech, and Signal Processing, vol. 2, pp. 401404, 2005.

[5] J. T. Rucker, J. E. Fowler, and N. H. Younan, "JPEG2000 Coding Strategies for Hyperspectral Data," Proceedings of the International Geoscience and Remote Sensing Symposium, Seoul, South Korea, vol. 1, pp. 128-131, July 2005.

[6] B. Penna, T. Tillo, E. Magli, and G. Olmo, "Progressive 3-D coding of hyperspectral images based on JPEG 2000," IEEE Geosciences and Remote Sensing Letters, vol. 3, no. 1, pp. 125-129, Jan. 2006.

[7] J. E. Fowler and J. T. Rucker, "3D Wavelet-Based Compression of Hyperspectral Imagery," in Hyperspectral Data Exploitation: Theory and Applications, C.-I. Chang, Ed., John Wiley \& Sons, Inc., Hoboken, NJ, 2007 (to appear).

[8] Q. Du and J. E. Fowler, "Hyperspectral image compression using JPEG2000 and principal components analysis," IEEE Geoscience and Remote Sensing Letters, vol. 4, no. 2, April 2007 (to appear).

[9] J. E. Dremmel, Applied Numerical Linear Algebra, Society for Industrial and Appled Mathematics, 1997.
[10] B. Penna, T. Tillo, E. Magli, and G. Olmo, "A new low complexity KLT for lossy hyperspectral data compression," Proceedings of IEEE Geoscience and Remote Sensing Symposium, 2006.

[11] K. I. Diamantaras and S. Y. Kung, Principal Component Analysis Neural Networks: Theory and Applications, John Wiley \& Sons, 1996.

[12] Q. Du and J. E. Fowler, "Low-complexity principal component analysis for hyperspectral image compression," International Journal of High Performance Computing Applications (in review).

[13] T. D. Sanger, "Optimal unsupervised learning in a singlelayer linear feedforward neural networks," Neural Networks, vol. 2 , no. 6 , pp. 459-473, 1989.

[14] M.-Y. Chiu, K.-B. Lee, and C.-W. Jen, "Optimal data transfer and buffering shemes for JPEG2000 encoder," Proceedings of IEEE Workshop on Signal Processing Systems, pp. 177-182, 2003.

[15] L. Liu, Z. Wang, N. Chen, and L. Zhang, "VLSI architecture of EBCOT Tier-2 encoder for JPEG2000," Proceedings of IEEE Workshop on Signal Processing Systems, pp. 225-228, 2005.

[16] G. Pastuszak, "A high-performance architecture for EBCOT in the JPEG2000 encoder," Proceedings of IEEE Workshop on Signal Processing Systems, pp. 693-698, 2005.

[17] D. B. Thomas and W. Luk, "High quality uniform random number generation for massively parallel simulations in FPGAs," Proceedings of the International Conference on Reconfigurable Computing and FPGAs, 2005.

[18] D. B. Thomas and W. Luk, "Efficient hardware generation of random variates with arbitrary distributions," Proceedings of the International Conference on Reconfigurable Computing and FPGAs, 2005.

TABLE I

SNR (IN DB) OF VARIOUS ALGORITHMS WITH DIFFERENT BIT RATES (IN BPPPB)

\begin{tabular}{|c|c|c|c|c|c|}
\hline Rate & 0.1 & 0.2 & 0.3 & 0.4 & 0.5 \\
\hline SubPCA+JPEG2000 & 38.55 & 42.31 & 44.43 & 45.76 & 46.69 \\
\hline PCA+JPEG2000 & 36.81 & 41.59 & 44.00 & 45.55 & 46.53 \\
\hline DWT+JPEG2000 & 29.00 & 33.21 & 35.82 & 37.69 & 39.31 \\
\hline 3D SPECK & 28.57 & 32.77 & 35.42 & 37.19 & 38.88 \\
\hline 3D SPIHT & 28.54 & 32.82 & 35.44 & 37.36 & 38.96 \\
\hline
\end{tabular}

TABLE II

SNR (IN DB) OF SUBPCA+JPEG2000 WITH SPATIAL DOWN-SAMPLING

\begin{tabular}{|c|c|c|c|c|c|}
\hline Rate & 0.1 & 0.2 & 0.3 & 0.4 & 0.5 \\
\hline SubPCA+JPEG2000 & 38.55 & 42.31 & 44.43 & 45.76 & 46.69 \\
\hline RDS_SubPCA+JPEG2000 & 38.55 & 42.27 & 44.36 & 45.62 & 46.52 \\
\hline RDS_SubPCA_NN+JPEG2000 & 38.52 & 42.23 & 44.30 & 45.59 & 46.51 \\
\hline UDS_SubPCA+JPEG2000 & 38.50 & 42.22 & 44.33 & 45.62 & 46.53 \\
\hline $\begin{array}{c}\text { UDS_SubPCA_NN+JPEG200 } \\
\overline{0}\end{array}$ & 38.50 & 42.21 & 44.30 & 45.58 & 46.50 \\
\hline
\end{tabular}


TABLE III

SNR (IN DB) OF SUBPCA+JPEG2000 WITH NON-ZERO MEAN DATA

\begin{tabular}{|c|c|c|c|c|c|}
\hline Rate & 0.1 & 0.2 & 0.3 & 0.4 & 0.5 \\
\hline SubPCA+JPEG2000 & 38.55 & 42.31 & 44.43 & 45.76 & 46.69 \\
\hline NZM_SubPCA+JPEG2000 & 37.93 & 42.07 & 44.29 & 45.67 & 46.60 \\
\hline NZM_RDS_SubPCA+JPEG2000 & 37.89 & 42.01 & 44.22 & 45.56 & 46.47 \\
\hline NZM_RDS_SubPCA_NN+JPEG2000 & 37.86 & 41.91 & 44.09 & 45.49 & 46.41 \\
\hline NZM_UDS_SubPCA+JPEG2000 & 37.88 & 41.97 & 44.15 & 45.52 & 46.43 \\
\hline NZM_UDS_SubPCA_NN+JPEG2000 & 37.87 & 41.88 & 44.07 & 45.49 & 46.41 \\
\hline
\end{tabular}

TABLE IV

SPECTAL FIDELITY (SPECTRAL ANGLE IN DEGREE) OF VARIOUS ALGORITHMS

\begin{tabular}{|c|c|c|c|c|c|c|}
\hline \multicolumn{2}{|l|}{ Rate } & 0.1 & 0.2 & 0.3 & 0.4 & 0.5 \\
\hline \multirow{3}{*}{$\begin{array}{c}\text { NZM_UDS_SubPCA_NN+JPEG200 } \\
0\end{array}$} & $\theta_{\mathrm{avg}}$ & $0.55^{\circ}$ & $0.35^{\circ}$ & $0.28^{\circ}$ & $0.24^{\circ}$ & $0.21^{\circ}$ \\
\hline & $\theta_{\text {peak }}$ & $8.30^{\circ}$ & $6.40^{\circ}$ & $6.25^{\circ}$ & $6.09^{\circ}$ & $5.81^{\circ}$ \\
\hline & $\theta_{\mathrm{std}}$ & $0.15^{\circ}$ & $0.09^{\circ}$ & $0.06^{\circ}$ & $0.05^{\circ}$ & $0.05^{\circ}$ \\
\hline \multirow{3}{*}{ UDS_SubPCA_NN+JPEG2000 } & $\theta_{\text {avg }}$ & $0.51^{\circ}$ & $0.34^{\circ}$ & $0.27^{\circ}$ & $0.23^{\circ}$ & $0.21^{\circ}$ \\
\hline & $\theta_{\text {peak }}$ & $7.05^{\circ}$ & $6.36^{\circ}$ & $6.23^{\circ}$ & $5.98^{\circ}$ & $5.47^{\circ}$ \\
\hline & $\theta_{\text {std }}$ & $0.13^{\circ}$ & $0.08^{\circ}$ & $0.06^{\circ}$ & $0.05^{\circ}$ & $0.05^{\circ}$ \\
\hline \multirow{3}{*}{ SubPCA+JPEG2000 } & $\theta_{\text {avg }}$ & $0.51^{\circ}$ & $0.34^{\circ}$ & $0.27^{\circ}$ & $0.23^{\circ}$ & $0.21^{\circ}$ \\
\hline & $\theta_{\text {peak }}$ & $2.92^{\circ}$ & $2.20^{\circ}$ & $1.76^{\circ}$ & $1.45^{\circ}$ & $1.23^{\circ}$ \\
\hline & $\theta_{\mathrm{std}}$ & $0.13^{\circ}$ & $0.08^{\circ}$ & $0.06^{\circ}$ & $0.05^{\circ}$ & $0.04^{\circ}$ \\
\hline \multirow{3}{*}{ PCA+JPEG2000 } & $\theta_{\mathrm{avg}}$ & $0.62^{\circ}$ & $0.37^{\circ}$ & $0.28^{\circ}$ & $0.24^{\circ}$ & $0.21^{\circ}$ \\
\hline & $\theta_{\text {peak }}$ & $2.91^{\circ}$ & $1.35^{\circ}$ & $0.86^{\circ}$ & $0.65^{\circ}$ & $0.54^{\circ}$ \\
\hline & $\theta_{\mathrm{std}}$ & $0.17^{\circ}$ & $0.09^{\circ}$ & $0.06^{\circ}$ & $0.05^{\circ}$ & $0.05^{\circ}$ \\
\hline \multirow{3}{*}{ DWT+JPEG2000 } & $\theta_{\text {avg }}$ & $1.47^{\circ}$ & $0.94^{\circ}$ & $0.70^{\circ}$ & $0.57^{\circ}$ & $0.48^{\circ}$ \\
\hline & $\theta_{\text {peak }}$ & $9.35^{\circ}$ & $5.11^{\circ}$ & $3.50^{\circ}$ & $2.76^{\circ}$ & 2.24 \\
\hline & $\theta_{\mathrm{std}}$ & $0.56^{\circ}$ & $0.32^{\circ}$ & $0.23^{\circ}$ & $0.17^{\circ}$ & $0.14^{\circ}$ \\
\hline \multirow{3}{*}{ 3D SPECK } & $\theta_{\text {avg }}$ & $1.56^{\circ}$ & $0.99^{\circ}$ & $0.74^{\circ}$ & $0.61^{\circ}$ & $0.50^{\circ}$ \\
\hline & $\theta_{\text {peak }}$ & $9.41^{\circ}$ & $5.33^{\circ}$ & $3.68^{\circ}$ & $2.42^{\circ}$ & $2.26^{\circ}$ \\
\hline & $\theta_{\mathrm{std}}$ & $0.59^{\circ}$ & $0.33^{\circ}$ & $0.24^{\circ}$ & $0.17^{\circ}$ & $0.15^{\circ}$ \\
\hline \multirow{3}{*}{ 3D SPIHT } & $\theta_{\text {avg }}$ & $1.56^{\circ}$ & $0.98^{\circ}$ & $0.73^{\circ}$ & $0.60^{\circ}$ & $0.50^{\circ}$ \\
\hline & $\theta_{\text {peak }}$ & $9.33^{\circ}$ & $5.24^{\circ}$ & $3.77^{\circ}$ & $2.46^{\circ}$ & $2.19^{\circ}$ \\
\hline & $\theta_{\text {std }}$ & $0.59^{\circ}$ & $0.32^{\circ}$ & $0.24^{\circ}$ & $0.17^{\circ}$ & $0.14^{\circ}$ \\
\hline
\end{tabular}

$\theta_{\text {avg }}$ is the mean value of spectral angles between the original and reconstructed pixel vectors;

$\theta_{\text {peak }}$ is the peak value of spectral angles between the original and reconstructed pixel vectors;

$\theta_{\text {std }}$ is the standard deviation of spectral angles between the original and reconstructed pixel vectors. 